\title{
Encephalopathy in an Elderly Patient Following Oral Ingestion of Topical Methyl Salicylate
}

\author{
Ji-Hyun Choi, MD ${ }^{1}$, Jin Park, MD ${ }^{1}$, Gyeong Seon Choi, MD ${ }^{1}$, Kee-Duk Park, MD, PhD ${ }^{1}$, \\ Kyoung-Gyu Choi, MD, $\mathrm{PhD}^{1}$, Young-Joo Lee, MD, $\mathrm{PhD}^{2}$, Jee-Hyang Jeong, MD, $\mathrm{PhD}^{1}$, \\ and Ji Young Yun, MD, $\mathrm{PhD}^{1}$ \\ 1 Department of Neurology, Ewha Womans University Mokdong Hospital, Ewha Womans University School of Medicine, Seoul, Korea \\ 2Department of Anesthesiology, Ewha Womans University Mokdong Hospital, Ewha Womans University School of Medicine, Seoul, Korea.
}

\begin{abstract}
Background: Methyl salicylate overdose is usually caused by frequent topical applications or accidental ingestion, especially in children. Case Report: A 77yearold woman was admitted with an altered mental status, mild fever, and tachypnea. Initial laboratory analysis showed metabolic acidosis with respiratory compensation without significant abnormal signs. On admission, hyperthermia was aggravated by antipyretics. On the second day after admission, one of her family members found methyl salicylate lotion in her cup, and she survived with hydration and conservative management.

Conclusion: We report the first case of an elderly patient intoxicated by oral ingestion of topical methyl salicylate.

J Neurocrit Care 2016;9(1):50-53
\end{abstract}

Key Words: Salicylate; Intoxication; Altered mental status; Hyperthermia

\section{INTRODUCTION}

Acetyl salicylate or methyl salicylate (liniment, Mentholatum ${ }^{\circledR}$ oil of wintergreen) are anti-analgesic and antirheumatic agents used as topical preparations for the skin world widely. Methyl salicylate, especially, can cause a rapid onset and severe form of salicylate toxicity from the liquid, highly concentrated, and lipid soluble forms. ${ }^{1}$ Methyl salicylate overdose is usually caused by frequent topical applications or accidental ingestion especially in children. ${ }^{2}$ Herein, we present an elderly patient who had an altered mental status and hyperthermia and was shown to be intoxicated from the oral ingestion of topical methyl

Received: March 14, 2016 / Revised: May 30, 2016

Accepted: June 7, 2016

Address for correspondence: Ji Young Yun, MD, PhD.

Department of Neurology, Ewha Womans University Mokdong Hospital, Ewha Womans University School of Medicine, 1071

AnYangCheon-ro, YangCheon-gu, Seoul 07985, Korea.

Tel: +82-2-2650-5289, Fax: +82-2-2650-2652

E-mail: dream-yoon@hanmail.net salicylate.

\section{CASE REPORT}

A 77-year-old woman was admitted with an altered mental status, mild fever with a temperature of $37.8^{\circ} \mathrm{C}$, and tachypnea with 24 breaths/minute. Her other vital signs were stable. She had a past history of hypertension, asthma and ischemic cerebral stroke one year ago. She had showed cognitive dysfunction since one and a half year ago, such as trouble in finding the way, forgetting the familiar names, and difficulties for keeping up personal hygiene.

On admission, her mental status was a stupor. Physical examination of the cardiac, pulmonary, abdominal, and neurological system was unremarkable. Initial laboratory analysis showed metabolic acidosis with respiratory compensation. Other laboratory findings for complete blood count, electrolyte, chemistry, urinalysis and chest $\mathrm{x}$-ray finding were unremarkable except for mild leukocytosis 

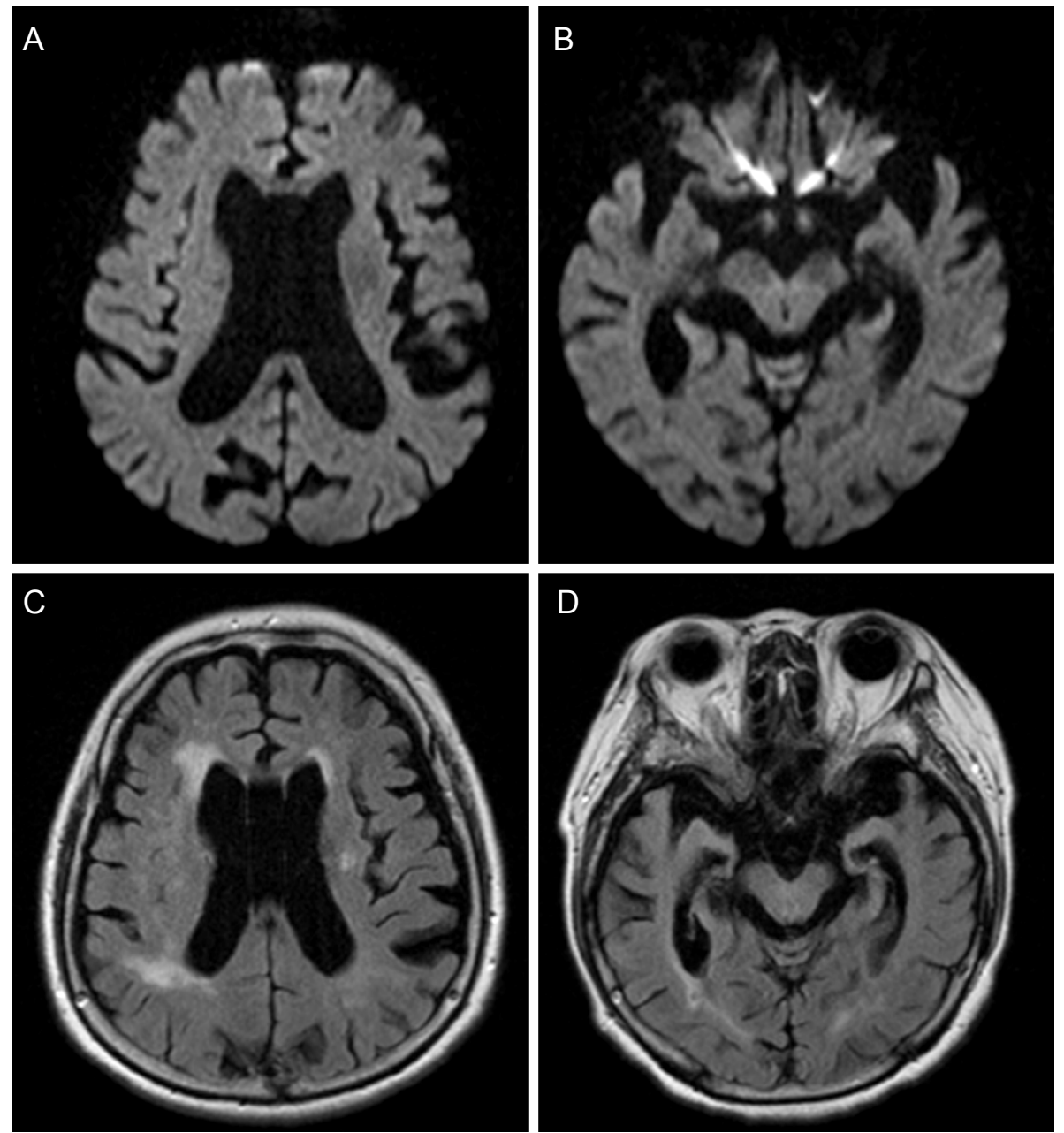

Figure 1. Brain magnetic resonance image. (A, B) Diffusion weighted imaging showed no acute ischemic lesion. (C, D) T2-weighted fluid attenuated inversion recovery showed nonspecific periventricular high signal intensities, old lacunar infarction in left basal ganglia, and diffuse senile brain atrophy.

at $17000 / \mathrm{uL}$ with a mildly elevated C-reactive protein at 1.6. There were no newly developed lesions on the brain magnetic resonance image (MRI) except for the known old lacunar infarction and diffuse senile atrophy (Fig. 1). Electroencephalography showed diffuse cerebral dysfunction without evidence of partial or generalized seizure. Cerebrospinal fluid analysis showed $0 / \mathrm{mm}^{3}$ white blood cells, $120 / \mathrm{mm}^{3}$ red blood cells related to traumatic tapping, 36 $\mathrm{mg} / \mathrm{dL}$ protein, $82 \mathrm{mg} / \mathrm{dL}$ glucose (ratio to serum glucose ratio is 0.55 ) with normal pressure.

Next day, on admission, her body temperature increased to $40.0^{\circ} \mathrm{C}$ and the hyperthermia was not controlled despite injection of an antipyretic agent and nonsteroidal antiinflammatory drugs (NSAIDs), and her mental status was still a stupor. That day, her family members found a cup with methyl salicylate lotion (Mentholatum ${ }^{\circledR}$ ) in her room and said she had had pica and memory dysfunction. Immediately, we stopped using NSAID as antipyretics and checked her salicylate concentration which was $325.8 \mathrm{ug} /$ $\mathrm{mL}$. After intravenous hydration and conservative management, she gradually improved to an alert status (Fig. 2).

\section{DISCUSSION}

Salicylate is commonly prescribed for its analgesic, antiinflammatory, antipyretic, and anti-platelet properties. There are many different forms of salicylate. Especially, liniments, herbal and Chinese topical preparations contain varying amounts of methyl salicylate as a topical anesthetic for pain in the muscles and joints. Because over- 
Body temperature $\left({ }^{\circ} \mathrm{C}\right)$

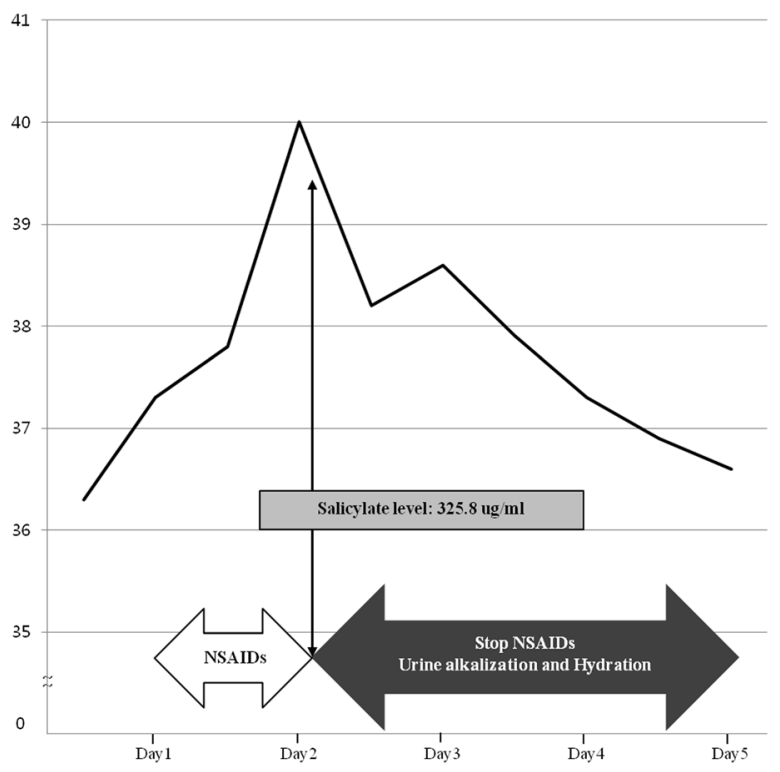

Figure 2. Hospital course of the patient: hyperthermia was aggravated by antipyretics and NSAIDs. When her family found a methyl salicylate lotion in her cup, her salicylate concentration was $325.8 \mathrm{ug} / \mathrm{mL}$. She survived with hydration and conservative management. NSAID, nonsteroidal anti-inflammatory drug.

the-counter methyl salicylate preparations come in liquid, highly concentrated, and lipid soluble forms, it could induce rapid and severe salicylate poisoning.

When compared to salicylate intoxication which is relatively easily diagnosed with a low mortality $(2 \%)$ in young adult, elderly people are likely to have a delayed diagnosed, and this delayed diagnosis is associated with a major morbidity and mortality of $30 \%$ and $25 \%$, respectively. ${ }^{3}$ Especially in topical methyl salicylate intoxication, oral ingestion usually occurs accidentally in children. ${ }^{2}$ And this is the first case of an elderly patient with topical salicylate intoxication caused by an accidental methyl salicylate overdose oral ingestion.

It was reported that $5 \mathrm{~mL}$ of oil of wintergreen is equal to $7000 \mathrm{mg}$ of salicylate, ${ }^{4}$ and the toxic dose of methyl salicylate is approximately $14 \mathrm{~mL}$ of Mentholatum ${ }^{\circledR}$, whereas for acetyl salicylate, it is $6.5 \mathrm{~g}$, (twenty $325 \mathrm{mg}$ aspirin tablets). ${ }^{5}$ A serum salicylate level of $300 \mathrm{mg} / \mathrm{L}$ following acute ingestion is potentially toxic. In the case presented here, the peripheral blood concentration of salicylic acid from methyl salicylate ingestion was $320 \mathrm{mg} / \mathrm{L}$ which was checked one day after admission. She manifested more aggravated hyperthermia with NSAIDs that was an intra- venous aspirin administration to control hyperthermia before we knew she was in a state of methyl salicylate intoxication. We believe that probably there was additional salicylate toxicity from the administered aspirin known as acetylsalicylic acid.

In methyl salicylate overdose, the hepatic metabolism diminishes due to limited capacity resulting in first-order elimination kinetics replaced by unpredictable, dose dependent, zero-order elimination. ${ }^{6}$ Toxic doses of salicylates are characterized by the stimulation of the respiratory center of the brain, uncoupling of oxidative phosphorylation, inhibition of Krebs cycle enzymes, alterations in lipid and amino acid metabolism, and electrolyte imbalance. ${ }^{7}$ In terms of salicylate toxicity on the central nervous system (CNS), it seems to directly stimulate the CNS respiratory center resulting in respiratory alkalosis accompanied by metabolic acidosis as a result of increased renal excretion of bicarbonates to compensate for the respiratory alkalosis. $^{8}$

Salicylate is known to be a toxin capable of uncoupling oxidative phosphorylation, which results in the loss of potential energy being released as heat manifesting hyperpyrexia. ${ }^{9}$ Hyperthermia is a sign that points to a fatal outcome in salicylate intoxication if not treated aggressively. ${ }^{10}$

In addition, glucose stores are depleted during salicylate intoxication because tissue glycolysis occurs including in the brain. This leads to a subsequent CNS glucose depletion even in the presence of normal blood glucose levels. ${ }^{8}$ CNS dysfunction manifests as disorientation, hallucination, irritability, and agitation as well as more serious symptoms including seizure, cerebral edema, or coma. ${ }^{7}$

The patient presented with an altered mental status and a fever above $40.0^{\circ} \mathrm{C}$.

However, there were no focal neurologic signs and significant abnormal findings except for metabolic acidosis with respiratory compensation. Because she manifested fever with acute mental change, cerebrospinal fluid analysis was initially examined despite no meningeal irritation signs and no infectious history recently. And it showed no abnormal findings with normal pressure, also no enhancing lesions on brain MRI. In fact, we did not suspect salicylate intoxication until her family member found a fluid smelling like methyl salicylate lotion in her cup on 
the second day after admission. Fortunately, blood salicylic acid level was not a lethal dose, and she survived with hydration and conservative management.

In conclusion, we report the first elderly patient who presented with an altered mental status and hyperthermia after oral ingestion of a topical salicylate. This case highlights the need to suspect salicylate intoxication when patients show a mental change from an unknown origin, acute respiratory alkalosis and metabolic acidosis without no significant abnormal signs, and hyperthermia aggravated by antipyretics especially in elderly patients with cognitive decline.

\section{Conflicts of interest}

The authors have no conflicts of interest to disclose.

\section{REFERENCES}

1. Chan TY. Potential dangers from topical preparations containing methyl salicylate. Hum Exp Toxicol 1996;15:74750.

2. Davis JE. Are one or two dangerous? Methyl salicylate ex- posure in toddlers. $J$ Eemerg Med 2007;32:63-9.

3. Anderson RJ, Potts DE, Gabow PA, Rumack BH, Schrier RW. Unrecognized adult salicylate intoxication. Ann Intern Med 1976;85:745-8.

4. Cauthen WL, Hester WH. Accidental ingestion of oil of wintergreen. J Fam Pract 1989;29:680-1.

5. Parker D, Martinez C, Stanley C, Simmons J, McIntyre IM. The analysis of methyl salicylate and salicylic acid from Chinese herbal medicine ingestion. J Anal Toxicol 2004;28:214-6.

6. Bari N. Salicylate poisoning. J Pak Med Assoc 1995;45:160-1.

7. Chyka PA, Erdman AR, Christianson G, Wax PM, Booze LL, Manoguerra AS, et al. Salicylate poisoning: an evidence-based consensus guideline for out-of-hospital management. Clin Toxicol (Phila) 2007;45:95-131.

8. Temple AR. Pathophysiology of aspirin overdosage toxicity, with implications for management. Pediatrics 1978;62(5 Pt 2 Suppl):873-6.

9. Eyer F, Zilker T. Bench-to-bedside review: mechanisms and management of hyperthermia due to toxicity. Crit Care 2007;11:236.

10. Gutknecht J. Salicylates and proton transport through lipid bilayer membranes: a model for salicylate-induced uncoupling and swelling in mitochondria. J Membr Biol 1990;115:253-60. 